\title{
Application of Optimal Homotopy Asymptotic Method to Doubly Wave Solutions of the Coupled Drinfel'd-Sokolov-Wilson Equations
}

\author{
H. Ullah, S. Islam, M. Idrees, and R. Nawaz \\ Department of Mathematics, Abdul Wali Khan University, Mardan, Pakistan \\ Correspondence should be addressed to H. Ullah; hakeemullah1@gmail.com
}

Received 7 August 2013; Accepted 5 November 2013

Academic Editor: Hai-Feng Huo

Copyright (C) $2013 \mathrm{H}$. Ullah et al. This is an open access article distributed under the Creative Commons Attribution License, which permits unrestricted use, distribution, and reproduction in any medium, provided the original work is properly cited.

The approximate solution of the doubly periodic wave solutions of the coupled Drinfel'd-Sokolov-Wilson equations has been considered by using the optimal homotopy asymptotic method (OHAM). We obtained the numerical solution of the problem and compared that with the OHAM solution. The obtained solutions show that OHAM is effective, simpler, easier, and explicit and gives a suitable way to control the convergence of the approximate solution.

\section{Introduction}

The coupled nonlinear partial differential equations (NPDEs) are widely used in applied mathematics, physics, and engineering sciences to offer the description of complex phenomena. Here we consider doubly periodic wave solutions of the coupled Drinfeld-Sokolov-Wilson equation of the form [1]

$$
\begin{gathered}
\frac{\partial u(x, t)}{\partial t}+3 v(x, t) \frac{\partial v(x, t)}{\partial x}=0, \\
\frac{\partial v(x, t)}{\partial t}+2 \frac{\partial^{3} v(x, t)}{\partial x^{3}} \\
+2 u(x, t) \frac{\partial v(x, t)}{\partial x}+\frac{\partial u(x, t)}{\partial x} v(x, t)=0,
\end{gathered}
$$

with

$$
u(x, 0)=x, \quad v(x, 0)=-x .
$$

The exact and explicit solution of the NPDEs in mathematical physics, engineering, and science plays an important role. The exact solution of NPDEs cannot be found easily as all NPDEs have infinitely many solutions. The analytical and exact solution of such problems is either not available in the literature or may be found by using transformation based on the invariance group analysis method [2], the Lie infinitesimal criterion [3], the symbolic computation [4], and the Backlund transformation [5]. All these methods reduced the complex equations into simple equations by using the transformation. In the literature most of the methods like the variational iterative method (VIM) [6], Adomian decomposition method (ADM) [7], differential transform method (DTM) [8], and homotopy perturbation method (HPM) [9] have been used for the solution of weakly NPDEs and few for strongly NPDEs. To tackle the strongly NPDEs the perturbation methods were introduced $[10,11]$. These methods contain a small parameter which cannot be found easily. New analytic methods such as the artificial parameters method [12], homotopy analysis method (HAM) [13], and homotopy perturbation method (HPM) [9] were introduced. These methods combined the homotopy with the perturbation techniques. Recently, Vasile Marinca et al. introduced OHAM [14-18] for the solution of nonlinear problems which made the perturbation methods independent of the assumption of small parameters and huge computational work.

The motivation of this paper is to boost OHAM for the solution of coupled NPDEs. In [19-24] OHAM has been proved to be valuable for obtaining an approximate solution of the single partial differential equation (PDE). Before these coupled NPDEs were not solved by OHAM. We have proved 
that OHAM is useful and reliable for NPDEs, showing its validity and great potential for the solution of transient physical phenomena in science and engineering.

In the succeeding section, the basic idea of OHAM is formulated for the solution of NPDEs. The effectiveness and efficiency of OHAM are shown in Section 3.

\section{Fundamental Mathematical Theory of OHAM}

Let us see the partial differential equation of the following form:

$$
\begin{gathered}
\mathscr{A}(u(x, t))+f(x, t)=0, \quad x \in \Omega, \\
\mathscr{B}\left(u, \frac{\partial u}{\partial x}\right)=0, \quad x \in \Gamma,
\end{gathered}
$$

where $\mathscr{A}$ is a differential operator, $u(x, t)$ is an unknown function, $x$ and $t$ denote spatial and temporal independent variables, respectively, $\Gamma$ is the boundary of $\Omega$, and $f(x, t)$ is a known analytic function. $\mathscr{A}$ can be divided into two parts $\mathscr{L}$ and $\mathcal{N}$ such that

$$
\mathscr{A}=\mathscr{L}+\mathscr{N}
$$

$\mathscr{L}$ is the simpler part of the partial differential equation which is easier to solve, and $\mathscr{N}$ contains the remaining part of $\mathscr{A}$.

According to OHAM, one can construct an optimal homotopy $\phi(x, t ; p): \Omega \times[0,1] \rightarrow \mathfrak{R}$ which satisfies

$$
\begin{aligned}
H(\phi(x, t ; p), p)= & (1-p)\{\mathscr{L}(\phi(x, t ; p))+f(x, t)\} \\
& -H(p)\{\mathscr{A}(\phi(x, t ; p))+f(x, t)\}=0,
\end{aligned}
$$

where the auxiliary function $H(p)$ is nonzero for $p \neq 0$ and $H(0)=0$. Equation (5) is called an optimal homotopy equation. Clearly, we have

$$
\begin{gathered}
p=0 \Longrightarrow H(\phi(x, t ; 0), 0)=\mathscr{L}(\phi(x, t ; 0))+f(x, t)=0, \\
p=1 \Longrightarrow H(\phi(x, t ; 1), 1) \\
=H(1)\{\mathscr{A}(\phi(x, t ; p))+f(x, t)\}=0 .
\end{gathered}
$$

Obviously, when $p=0$ and $p=1$ we obtain

$$
\phi(x, t ; 0)=u_{0}(x, t), \quad \phi(x, t ; 1)=u(x, t),
$$

respectively. Thus, as $p$ varies from 0 to 1 , the solution $\phi(x, t ; p)$ approaches from $u_{0}(x, t)$ to $u(x, t)$, where $u_{0}(x, t)$ is obtained from (5) for $p=0$ :

$$
\mathscr{L}\left(u_{0}(x, t)\right)+f(x, t)=0, \quad \mathscr{B}\left(u_{0}, \frac{\partial u_{0}}{\partial x}\right)=0 .
$$

Next, we choose the auxiliary function $H(p)$ in the form

$$
H(p)=p C_{1}+p^{2} C_{2}+\cdots
$$

To get an approximate solution, we expand $\phi\left(x, t ; p, C_{i}\right)$ by Taylor's series about $p$ in the following manner:

$$
\begin{array}{r}
\phi\left(x, t ; p, C_{i}\right)=u_{0}(x, t)+\sum_{k=1}^{\infty} u_{k}\left(x, t ; C_{i}\right) p^{k} \\
i=1,2, \ldots
\end{array}
$$

Substituting (10) into (5) and equating the coefficient of the like powers of $p$, we obtain the zeroth order problem, given by (8), the first and second order problems are given by (11)-(12), respectively, and the general governing equations for $u_{k}(x, t)$ are given by (13):

$$
\begin{aligned}
& \mathscr{L}\left(u_{1}(x, t)\right)=C_{1} \mathcal{N}_{0}\left(u_{0}(x, t)\right), \quad \mathscr{B}\left(u_{1}, \frac{\partial u_{1}}{\partial x}\right)=0 \\
& \mathscr{L}\left(u_{2}(x, t)\right)-\mathscr{L}\left(u_{1}(x, t)\right) \\
& =C_{2} \mathcal{N}_{0}\left(u_{0}(x, t)\right) \\
& +C_{1}\left[\mathscr{L}\left(u_{1}(x, t)\right)+\mathcal{N}_{1}\left(u_{0}(x, t), u_{1}(x, t)\right)\right] \\
& \qquad \mathscr{B}\left(u_{2}, \frac{\partial u_{2}}{\partial x}\right)=0, \\
& \mathscr{L}\left(u_{k}(x, t)\right)-\mathscr{L}\left(u_{k-1}(x, t)\right) \\
& =C_{k} \mathcal{N}_{0}\left(u_{0}(x, t)\right) \\
& +\sum_{i=1}^{k-1} C_{i}\left[\mathscr{L}\left(u_{k-i}(x, t)\right)\right. \\
& \left.+\mathcal{N}_{k-i}\left(u_{0}(x, t), u_{1}(x, t), \ldots, u_{k-i}(x, t)\right)\right] \\
& k=2,3, \ldots, \quad \mathscr{B}\left(u_{k}, \frac{\partial u_{k}}{\partial x}\right)=0,
\end{aligned}
$$

where $\mathcal{N}_{k-i}\left(u_{0}(x, t), u_{1}(x, t), \ldots, u_{k-i}(x, t)\right)$ are the coefficients of $p^{k-i}$ in the expansion of $\mathcal{N}(\phi(x, t ; p))$ about the embedding parameter $p$ :

$$
\begin{aligned}
\mathcal{N} & \left(\phi\left(x, t ; p, C_{i}\right)\right) \\
& =\mathcal{N}_{0}\left(u_{0}(x, t)\right)+\sum_{k \geq 1} \mathcal{N}_{k}\left(u_{0}, u_{1}, u_{2}, \ldots, u_{k}\right) p^{k} .
\end{aligned}
$$

It should be underscored that the $u_{k}$ for $k \geq 0$ are governed by the linear equations with linear boundary conditions that come from the original problem, which can be easily solved.

It has been observed that the convergence of the series equation (10) depends upon the auxiliary constants $C_{1}, C_{2}, \ldots$. If it is convergent at $p=1$, one has

$$
\widetilde{u}\left(x, t ; C_{i}\right)=u_{0}(x, t)+\sum_{k \geq 1} u_{k}\left(x, t ; C_{i}\right)
$$


Substituting (15) into (1), it results the following expression for the residual:

$$
R\left(x, t ; C_{i}\right)=\mathscr{L}\left(\widetilde{u}\left(x, t ; C_{i}\right)\right)+f(x, t)+\mathcal{N}\left(\widetilde{u}\left(x, t ; C_{i}\right)\right) .
$$

In actual computation $k=1,2,3, \ldots, m$.

If $R\left(x, t ; C_{i}\right)=0$, then $\tilde{u}\left(x, t ; C_{i}\right)$ is the exact solution of the problem. Generally it does not happen, especially in nonlinear problems.

For the determinations of auxiliary constants, $C_{i}, i=$ $1,2, \ldots, m$, there are different methods like Galerkin's method, the Ritz method, the least squares method, and, the collocation method. One can apply the method of least squares as under

$$
\begin{gathered}
J\left(C_{i}\right)=\int_{0}^{t} \int_{\Omega} R^{2}\left(x, t ; C_{i}\right) d x d t \\
\frac{\partial J}{\partial C_{1}}=\frac{\partial J}{\partial C_{2}}=\cdots=\frac{\partial J}{\partial C_{m}}=0 .
\end{gathered}
$$

The $m$ th order approximation can be obtained by these constants. The constants $C_{i}$ can also be determined by another method as under

$$
\begin{array}{r}
R\left(h_{1} ; C_{i}\right)=R\left(h_{2} ; C_{i}\right)=\cdots=R\left(h_{m} ; C_{i}\right)=0, \\
i=1,2, \ldots, m,
\end{array}
$$

at any time $t$, where $h_{i} \in \Omega$.

The more general auxiliary function $H(p)$ is useful for convergence, which depends upon constants $C_{1}, C_{2}, \ldots$, can be optimally identified by (18), and is useful in error minimization.

\section{Application of OHAM to Doubly Periodic Wave Solutions of the Coupled Drinfel'd-Sokolov-Wilson Equation}

To demonstrate the effectiveness of the extended formulation of OHAM for coupled nonlinear partial differential equations (NPDEs), we consider the doubly periodic wave solutions of the coupled Drinfel'd-Sokolov-Wilson equations (1) with the boundary condition (2).
Applying the method formulated in Section 2 leads to the following:

$$
\begin{aligned}
& \mathscr{L}(u(x, t))=\frac{\partial u(x, t)}{\partial t}, \quad \mathcal{N}(v(x, t))=3 v(x, t) \frac{\partial v(x, t)}{\partial x}, \\
& \mathscr{L}(v(x, t))=\frac{\partial v(x, t)}{\partial t}, \\
& \mathcal{N}(u, v)=2 \frac{\partial^{3} v(x, t)}{\partial x^{3}}+2 u(x, t) \frac{\partial v(x, t)}{\partial x}+\frac{\partial u(x, t)}{\partial x} v(x, t) \\
& (1-p) \frac{\partial u(x, t)}{\partial t} \\
& -H_{1}(p)\left[\frac{\partial u(x, t)}{\partial t}+3 v(x, t) \frac{\partial v(x, t)}{\partial t}\right]=0, \\
& (1-p) \frac{\partial v(x, t)}{\partial t} \\
& -H_{2}(p)\left[\begin{array}{c}
\frac{\partial u(x, t)}{\partial t}+2 \frac{\partial^{3} v(x, t)}{\partial x^{3}}+2 u(x, t) \frac{\partial v(x, t)}{\partial x} \\
+\frac{\partial u(x, t)}{\partial x} v(x, t)
\end{array}\right] \\
& =0 \text {. }
\end{aligned}
$$

We consider

$$
\begin{gathered}
u=u_{0}+p u_{1}+p^{2} u_{2}, \\
v=v_{0}+p v_{1}+p^{2} v_{2}, \\
H_{1}(p)=p C_{11}+p^{2} C_{12}, \\
H_{2}(p)=p C_{21}+p^{2} C_{22} .
\end{gathered}
$$

3.1. Zeroth Order System. We have

$$
\begin{aligned}
& \frac{\partial u_{0}(x, t)}{\partial t}=0, \\
& \frac{\partial v_{0}(x, t)}{\partial t}=0,
\end{aligned}
$$

with initial conditions

$$
u_{0}(x, 0)=x, \quad v_{0}(x, 0)=-x .
$$

Its solution

$$
u_{0}(x, t)=x, \quad v_{0}(x, t)=-x .
$$


3.2. First Order System. We have

$$
\begin{gathered}
\frac{\partial u_{1}(x, t)}{\partial t}=\left(1+C_{11}\right) \frac{\partial u_{0}(x, t)}{\partial t}+3 C_{11} v_{0}(x, t) \frac{\partial v_{0}(x, t)}{\partial x}, \\
\frac{\partial v_{1}(x, t)}{\partial t} \\
=\left\{\frac{\partial v_{0}(x, t)}{\partial t}+C_{21} v_{0}(x, t)\left(1+\frac{\partial u_{0}(x, t)}{\partial t}\right)\right. \\
\left.+2 C_{21} u_{0}(x, t) \frac{\partial v_{0}(x, t)}{\partial x}+2 C_{21} \frac{\partial^{3} v_{0}(x, t)}{\partial x^{3}}\right\}, \\
u_{1}(x, 0)=0, \quad v_{1}(x, 0)=0 .
\end{gathered}
$$

Its solutions

$$
\begin{gathered}
u_{1}(x, t)=3 t x C_{11}, \\
v_{1}(x, t)=-t\left(x+4 x C_{21}\right) .
\end{gathered}
$$

\subsection{Second Order System. We have}

$$
\begin{aligned}
& \frac{\partial u_{2}(x, t)}{\partial t} \\
& =\left\{\left(1+C_{11}\right) \frac{\partial u_{1}(x, t)}{\partial t}+C_{21} \frac{\partial u_{0}(x, t)}{\partial x}\right. \\
& +3\left(C_{12} v_{0}(x, t)+C_{11} v_{1}(x, t)\right) \frac{\partial v_{0}(x, t)}{\partial x} \\
& \left.+3 C_{11} v_{0}(x, t) \frac{\partial v_{1}(x, t)}{\partial x}\right\}, \\
& \frac{\partial v_{2}(x, t)}{\partial t} \\
& =\left\{\frac{\partial v_{1}(x, t)}{\partial t}+C_{21} v_{1}(x, t)+C_{22} v_{0}(x, t)\right. \\
& +\left(C_{22} v_{0}(x, t)+C_{21} v_{1}(x, t)\right) \\
& \times \frac{\partial u_{0}(x, t)}{\partial x}+2\left(C_{22} u_{0}(x, t)+C_{21} u_{1}(x, t)\right) \frac{\partial v_{0}(x, t)}{\partial x} \\
& +2 C_{21} u_{0}(x, t) \frac{\partial v_{1}(x, t)}{\partial x} \\
& \left.+2 C_{22} \frac{\partial^{3} v_{0}(x, t)}{\partial x^{3}}+2 C_{21} \frac{\partial^{3} v_{1}(x, t)}{\partial x^{3}}\right\},
\end{aligned}
$$

with

$$
u_{2}(x, 0)=0, \quad v_{2}(x, 0)=0 .
$$

Its solutions

$$
\begin{aligned}
& u_{2}(x, t)=\frac{3}{2}\left[C_{11}(1+2 x) t^{2}+2 C_{11}^{2} x t(1+4 t)+C_{12} x t\right] \\
& v_{2}(x, t)=\frac{1}{2}[ \\
& \quad-2 x t-4 C_{21} x t(2+t) \\
& \left.\quad-9 C_{11} C_{21} x t^{2}-16 C_{21}^{2} x t^{2}-8 C_{22} x t\right] .
\end{aligned}
$$

Adding (26), (29), and (28), we obtain

$$
\begin{aligned}
u(x, t)= & x+3 t x C_{11} \\
& +\frac{3}{2}\left[C_{11}(1+2 x) t^{2}+2 C_{11}^{2} x t(1+4 t)+C_{12} x t\right] \\
v(x, t)= & -x-t\left(x+4 x C_{21}\right) \\
+ & \frac{1}{2}\left[-2 x t-4 C_{21} x t(2+t)\right. \\
& \left.\quad-9 C_{11} C_{21} x t^{2}-16 C_{21}^{2} x t^{2}-8 C_{22} x t\right] .
\end{aligned}
$$

For the calculations of the constants $C_{11}, C_{12}, C_{21}$, and $C_{22}$ using (30) in (1) and applying the procedure mentioned in (16)-(19), we get

$$
\begin{gathered}
C_{11}=0, \quad C_{12}=-0.6282197208603675, \\
C_{21}=0.3177122963169145, \\
C_{22}=-1.689990604397596, \\
u(x, t)=x-1.88466 x t, \\
v(x, t)=-x-2.27085 x t+\frac{1}{2}\left(8.97823 x t-2.88591 x t^{2}\right) .
\end{gathered}
$$

\section{Results and Discussions}

The formulation presented in Section 2 provides highly accurate solutions for the problems demonstrated in Section 3. We have used Mathematica 7 for most of our computational work. In Tables 1 and 3, we have presented absolute errors for $u(x, t)$ and $v(x, t)$ at a spatial domain $[0,0.4]$ for $t=0.01$, $t=0.015, t=0.1, t=0.2, t=0.3$, and $t=0.4$, while in Tables 2 and 4 the convergence of the OHAM solution is given, through first and second order absolute errors at time $t=0.1$ and $0 \leq x \leq 1$. Here we observe that the OHAM solution converges rapidly with increasing order of approximation. From Tables 1-4 and Figures 1, 2, 3, 4, 5, 6, 7, 8, 9, and 10 it is evident that the OHAM results are nearly identical to the numerical results. Here the results are very consistent with the increasing time.

\section{Conclusion}

In this paper, we have seen the effectiveness of OHAM [16-20] in doubly periodic wave solutions of the coupled 
TABLE 1: Absolute error of OHAM solution of $u(x, t)$ corresponding to the numerical solution.

\begin{tabular}{cccccc}
\hline$x$ & & & $t$ & & \\
$t=0.3$ & $t=0.4$ \\
\hline 0.1 & $4.10634 \times 10^{-6}$ & $4.10654 \times 10^{-5}$ & $5.52825 \times 10^{-6}$ & $3.36769 \times 10^{-6}$ & $1.28783 \times 10^{-6}$ \\
0.2 & $1.83012 \times 10^{-6}$ & $7.75201 \times 10^{-5}$ & $2.39901 \times 10^{-6}$ & $4.39072 \times 10^{-6}$ & $1.49213 \times 10^{-6}$ \\
0.3 & $4.78801 \times 10^{-6}$ & $4.78851 \times 10^{-5}$ & $7.84255 \times 10^{-6}$ & $1.46108 \times 10^{-6}$ & $1.06140 \times 10^{-6}$ \\
0.4 & $4.90183 \times 10^{-6}$ & $2.39950 \times 10^{-6}$ & $6.03249 \times 10^{-6}$ & $7.54685 \times 10^{-6}$ & $1.23535 \times 10^{-6}$ \\
0.5 & $6.43592 \times 10^{-6}$ & $6.58079 \times 10^{-6}$ & $2.84924 \times 10^{-6}$ & $1.11220 \times 10^{-6}$ & $3.14244 \times 10^{-6}$ \\
0.6 & $7.97001 \times 10^{-6}$ & $8.11025 \times 10^{-6}$ & $2.85555 \times 10^{-6}$ & $7.02914 \times 10^{-6}$ & $9.25554 \times 10^{-6}$ \\
0.7 & $4.95914 \times 10^{-6}$ & $4.95414 \times 10^{-6}$ & $6.48274 \times 10^{-6}$ & $9.22908 \times 10^{-7}$ & $4.49099 \times 10^{-7}$ \\
0.8 & $6.03249 \times 10^{-7}$ & $1.49270 \times 10^{-6}$ & $3.29949 \times 10^{-6}$ & $5.25885 \times 10^{-7}$ & $6.18914 \times 10^{-7}$ \\
0.9 & $5.59132 \times 10^{-7}$ & $6.83350 \times 10^{-6}$ & $2.27285 \times 10^{-7}$ & $7.90433 \times 10^{-7}$ & $1.41393 \times 10^{-7}$ \\
1.0 & $2.84925 \times 10^{-7}$ & $2.17437 \times 10^{-7}$ & $6.93298 \times 10^{-7}$ & $1.00563 \times 10^{-7}$ & $4.59537 \times 10^{-8}$ \\
\hline
\end{tabular}

TABLE 2: Comparison of first order and second order errors of $u(x, t)$ corresponding to the numerical solution at time $t=0.1$, and $0 \leq x \leq 1$.

\begin{tabular}{lcc}
\hline$x$ & First order absolute error & Second order absolute error \\
\hline 0.1 & $1.60818 \times 10^{-2}$ & $4.10654 \times 10^{-5}$ \\
0.2 & $3.39636 \times 10^{-2}$ & $7.75201 \times 10^{-5}$ \\
0.3 & $5.18454 \times 10^{-2}$ & $4.78851 \times 10^{-5}$ \\
0.4 & $6.97272 \times 10^{-2}$ & $2.39950 \times 10^{-6}$ \\
0.5 & $8.76090 \times 10^{-2}$ & $6.58079 \times 10^{-6}$ \\
0.6 & $1.05491 \times 10^{-1}$ & $8.11025 \times 10^{-6}$ \\
0.7 & $1.23373 \times 10^{-1}$ & $4.95414 \times 10^{-6}$ \\
0.8 & $1.41254 \times 10^{-1}$ & $1.49270 \times 10^{-6}$ \\
0.9 & $1.59136 \times 10^{-1}$ & $6.83350 \times 10^{-6}$ \\
1.0 & $1.77018 \times 10^{-1}$ & $2.17437 \times 10^{-7}$ \\
\hline
\end{tabular}

TABLE 3: Absolute error of OHAM solution of $v(x, t)$ corresponding to the numerical solution.

\begin{tabular}{cccccc}
\hline$x$ & & & $t$ & & \\
& $t=0.01$ & $t=0.1$ & $t=0.2$ & $t=0.3$ & -0.4 \\
\hline 0.1 & $-8.38255 \times 10^{-5}$ & $-7.99845 \times 10^{-6}$ & $-8.50767 \times 10^{-5}$ & $-1.17209 \times 10^{-4}$ & $-1.25237 \times 10^{-3}$ \\
0.2 & $-4.70697 \times 10^{-5}$ & $-8.90496 \times 10^{-6}$ & $-1.58744 \times 10^{-5}$ & $-2.24198 \times 10^{-4}$ & $-3.79536 \times 10^{-3}$ \\
0.3 & $-1.19722 \times 10^{-5}$ & $-1.34145 \times 10^{-6}$ & $-9.61422 \times 10^{-5}$ & $-1.02833 \times 10^{-4}$ & $-6.03144 \times 10^{-3}$ \\
0.4 & $-1.94189 \times 10^{-5}$ & $-9.51779 \times 10^{-6}$ & $-1.93986 \times 10^{-5}$ & $-1.02833 \times 10^{-4}$ & $-8.46213 \times 10^{-3}$ \\
0.5 & $-4.28514 \times 10^{-5}$ & $-1.08137 \times 10^{-5}$ & $-2.03181 \times 10^{-5}$ & $-1.01524 \times 10^{-4}$ & $-1.40224 \times 10^{-3}$ \\
0.6 & $-1.15699 \times 10^{-5}$ & $-7.64659 \times 10^{-5}$ & $-1.78514 \times 10^{-5}$ & $-9.06291 \times 10^{-3}$ & $-6.36149 \times 10^{-3}$ \\
0.7 & $-7.91541 \times 10^{-4}$ & $-9.20329 \times 10^{-5}$ & $-2.25841 \times 10^{-4}$ & $-6.88889 \times 10^{-3}$ & $-1.54670 \times 10^{-2}$ \\
0.8 & $-1.50929 \times 10^{-4}$ & $-6.25928 \times 10^{-5}$ & $-9.04686 \times 10^{-4}$ & $-8.30865 \times 10^{-2}$ & $-9.92225 \times 10^{-2}$ \\
0.9 & $-1.12584 \times 10^{-4}$ & $-1.06202 \times 10^{-4}$ & $-2.26639 \times 10^{-4}$ & $-7.63087 \times 10^{-2}$ & $-8.63452 \times 10^{-1}$ \\
1.0 & $-7.34287 \times 10^{-4}$ & $-2.96571 \times 10^{-4}$ & $-5.29813 \times 10^{-3}$ & $-1.67205 \times 10^{-2}$ & $-1.34788 \times 10^{-1}$ \\
\hline
\end{tabular}

TABLE 4: Comparison of first order and second order errors of $v(x, t)$ corresponding to the numerical solution at time $t=0.1$, and $0 \leq x \leq 1$.

\begin{tabular}{lcc}
\hline$x$ & First order absolute error & Second order absolute error \\
\hline 0.1 & $5.67234 \times 10^{-2}$ & $2.25631 \times 10^{-6}$ \\
0.2 & $1.13447 \times 10^{-2}$ & $1.58864 \times 10^{-6}$ \\
0.3 & $1.70170 \times 10^{-1}$ & $2.38296 \times 10^{-6}$ \\
0.4 & $2.26894 \times 10^{-1}$ & $3.17721 \times 10^{-6}$ \\
0.5 & $2.83617 \times 10^{-1}$ & $2.91721 \times 10^{-5}$ \\
0.6 & $3.40341 \times 10^{-1}$ & $1.25812 \times 10^{-5}$ \\
0.7 & $3.97064 \times 10^{-1}$ & $2.50126 \times 10^{-5}$ \\
0.8 & $4.53787 \times 10^{-1}$ & $1.28317 \times 10^{-4}$ \\
0.9 & $5.10510 \times 10^{-1}$ & $1.29810 \times 10^{-4}$ \\
1.0 & $5.67234 \times 10^{-1}$ & $2.94185 \times 10^{-4}$ \\
\hline
\end{tabular}




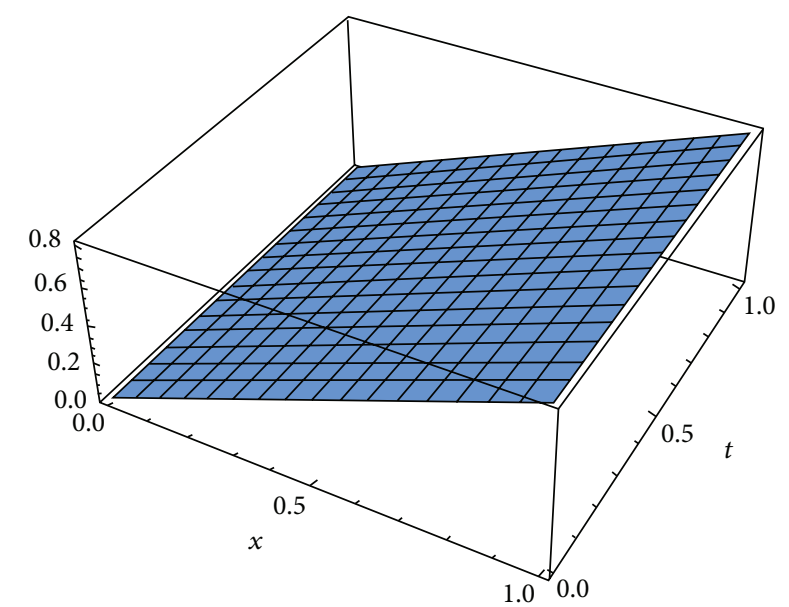

FIGURE 1: 3D approximate solution of $u(x, t)$ for $t=0.1$.

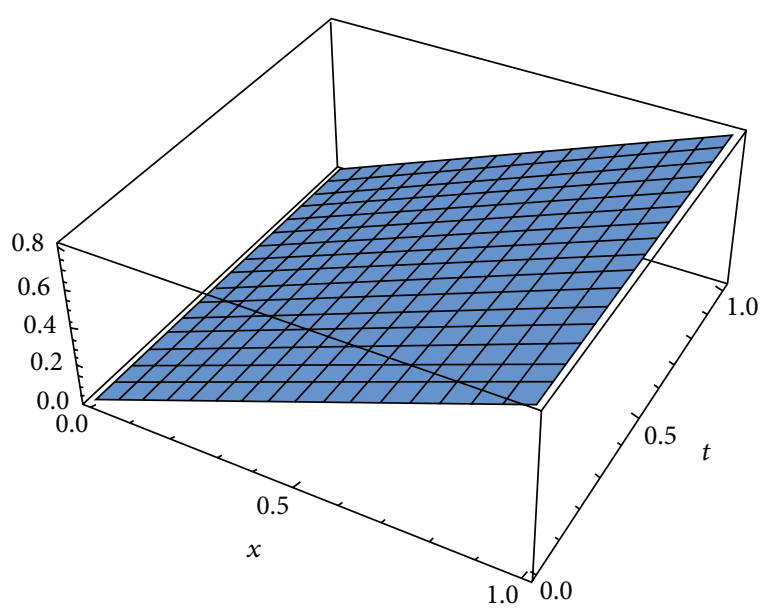

FIGURE 2: 3D numerical solution of $u(x, t)$ for $t=0.1$.

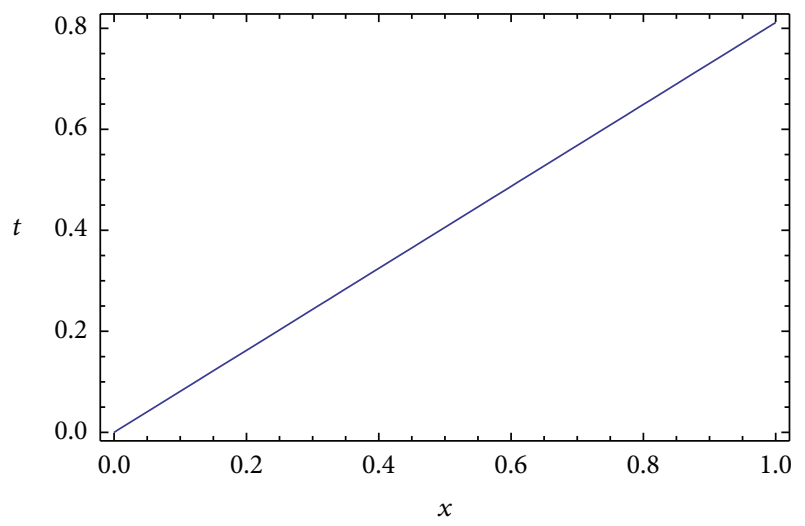

FIGURE 3: 2D approximate solution of $u(x, t)$ for $t=0.1$.

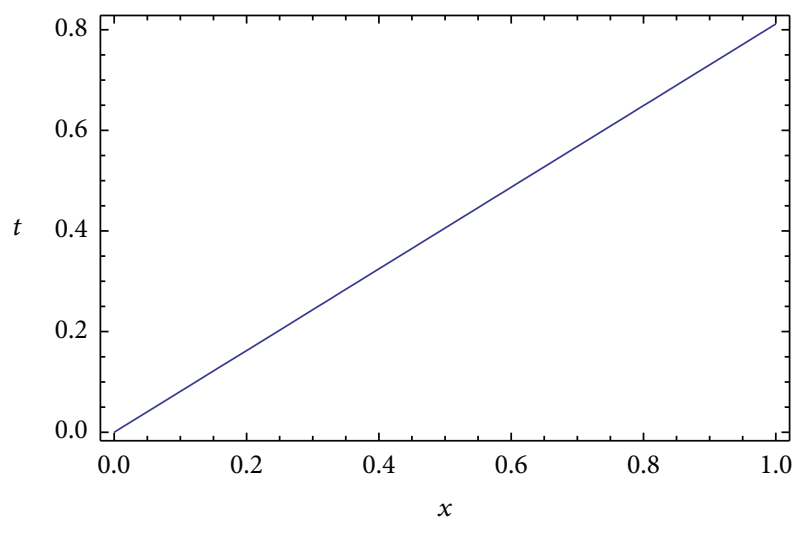

FIgURE 4: 2D numerical solution for $t=0.1$.

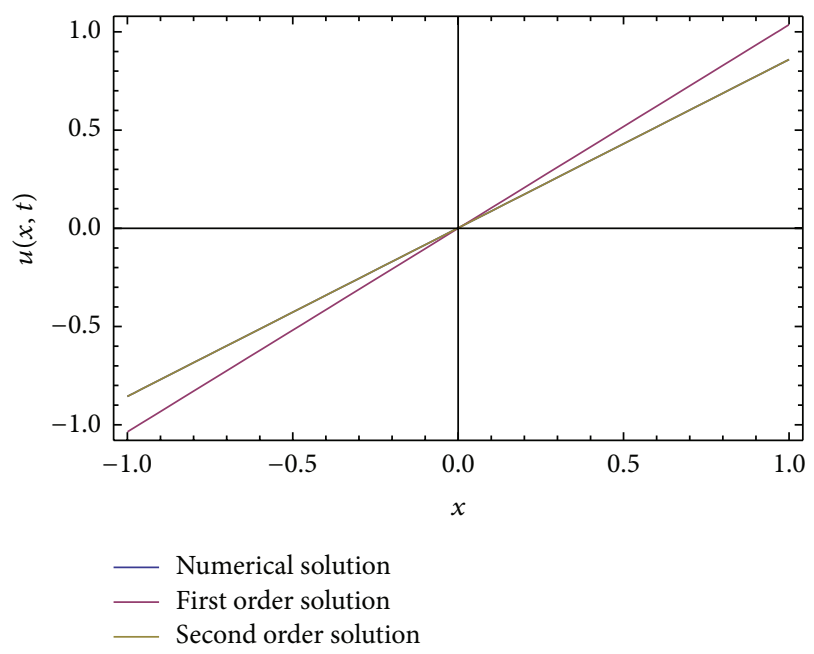

FIGURE 5: 2D numerical, zeroth, first, and second order solutions of $u(x, t)$ for $t=0.5$.

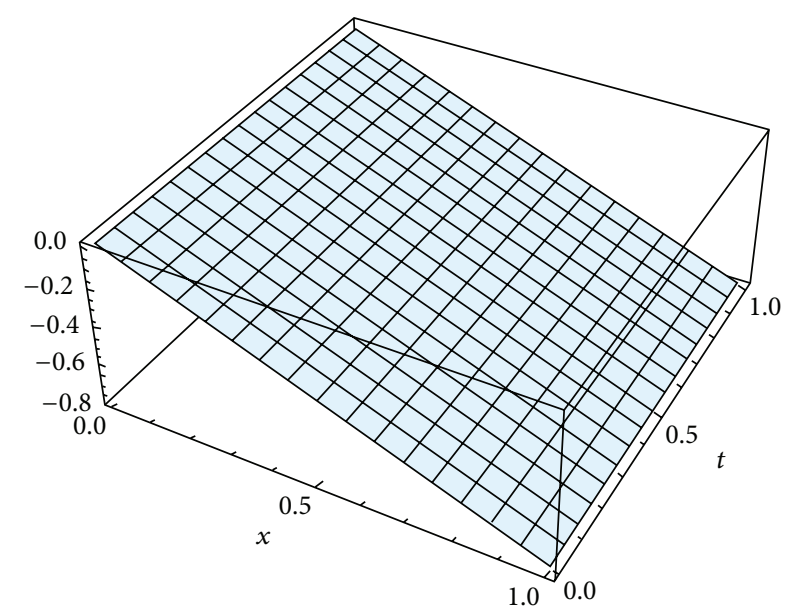

FIgURE 6: 3D approximate solution of $v(x, t)$ for $t=0.1$. 


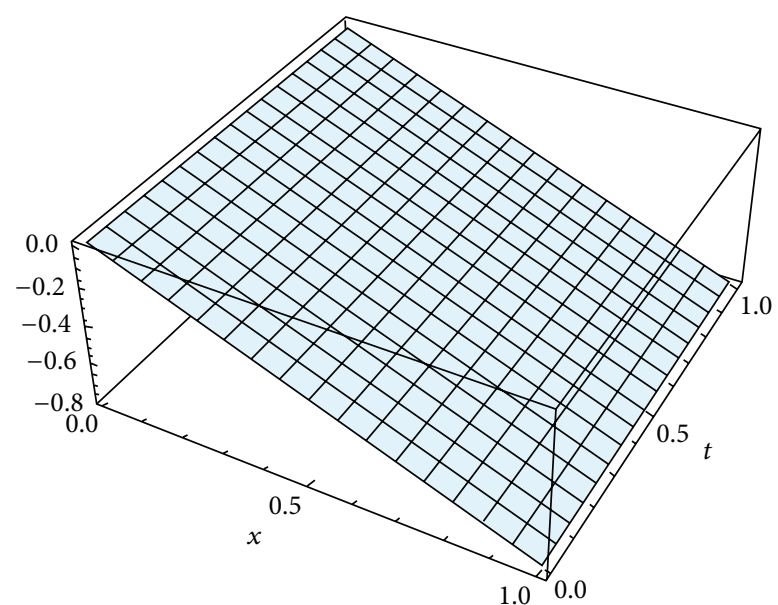

Figure 7: 3D exact solution of $v(x, t)$ for $t=0.1$.

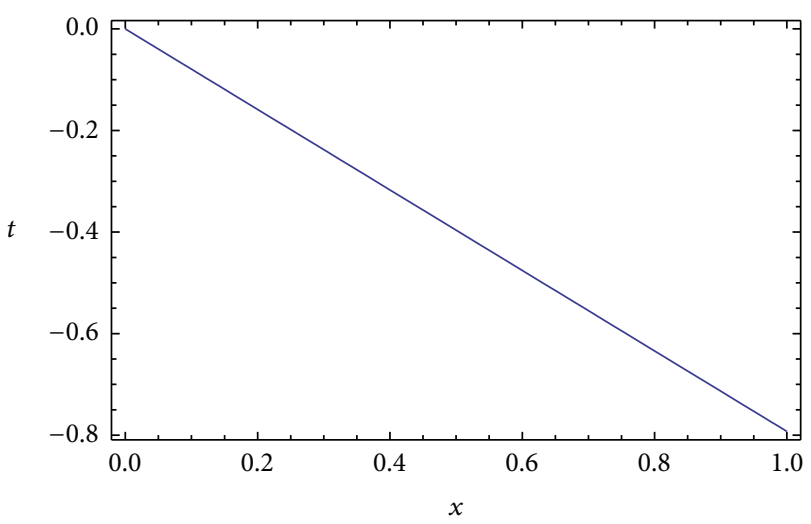

FIGURE 8: 2D approximate solution of $v(x, t)$ for $t=0.1$.

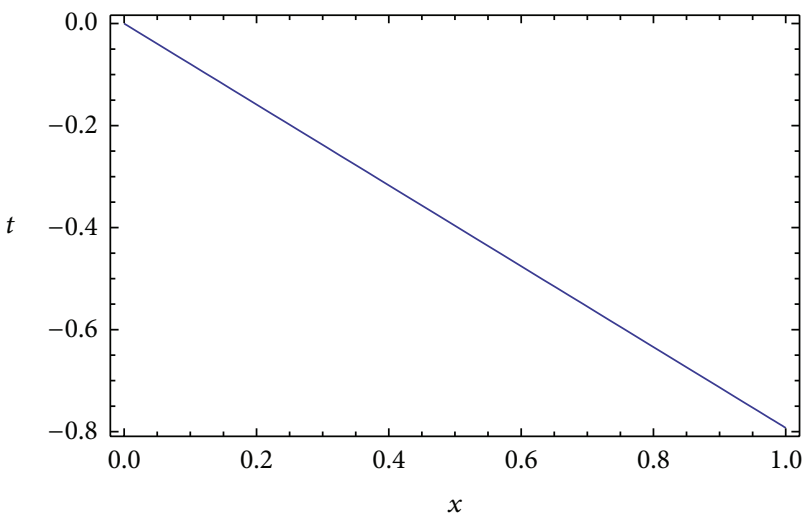

FIGURE 9: 2D numerical solution of $v(x, t)$ for $t=0.1$.

Drinfel'd-Sokolov-Wilson equation. By applying the basic idea of OHAM to doubly periodic wave solutions of the coupled Drinfel'd-Sokolov-Wilson equation, we found it simpler in applicability, more convenient to control convergence, and involving less computational overhead. Therefore, OHAM

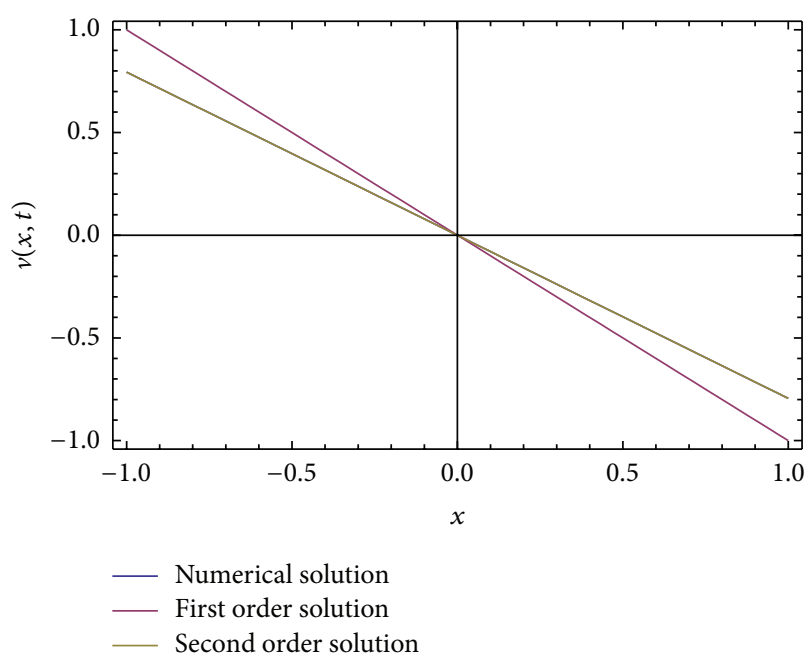

FIGURE 10: 2D numerical, zeroth, first, and second order solution of $v(x, t)$ for $t=0.5$.

shows its validity and great potential for the solution of time dependant problems in science and engineering.

\section{Conflict of Interests}

The authors declare that there is no conflict of interests regarding the publication of this paper.

\section{References}

[1] M. Inc, "On numerical doubly periodic wave solutions of the coupled Drinfel'd-Sokolov-Wilson equation by the decomposition method," Applied Mathematics and Computation, vol. 172, no. 1, pp. 421-430, 2006.

[2] M. Torrisi, R. Tracinà, and A. Valenti, "A group analysis approach for a nonlinear differential system arising in diffusion phenomena," Journal of Mathematical Physics, vol. 37, no. 9, pp. 4758-4767, 1996.

[3] P. G. Drzain and R. S. Johnson, An Introduction Discussion the Theory of Solution and Its Diverse Applications, Cambridge University Press, Cambridge, Mass, USA, 1989.

[4] B. Abdel-Hamid, "Exact solutions of some nonlinear evolution equations using symbolic computations," Computers and Mathematics with Applications, vol. 40, no. 2, pp. 291-302, 2000.

[5] G. Bluman and S. Kumei, "On the remarkable nonlinear diffusion equation $(\partial / \partial \mathrm{x})\left[\mathrm{a}(\mathrm{u}+\mathrm{b})^{-2}(\partial \mathrm{u} / \partial \mathrm{x})\right]-(\partial \mathrm{u} / \partial \mathrm{t})=0$," Journal of Mathematical Physics, vol. 21, no. 5, pp. 1019-1023, 1980.

[6] C. Chun, "Fourier-series-based variational iteration method for a reliable treatment of heat equations with variable coefficients," International Journal of Nonlinear Sciences and Numerical Simulation, vol. 10, no. 11-12, pp. 1383-1388, 2009.

[7] S. H. Chowdhury, "A comparison between the modified homotopy perturbation method and adomian decomposition method for solving nonlinear heat transfer equations," Journal of Applied Sciences, vol. 11, no. 8, pp. 1416-1420, 2011.

[8] H. Yaghoobi and M. Torabi, "The application of differential transformation method to nonlinear equations arising in heat transfer," International Communications in Heat and Mass Transfer, vol. 38, no. 6, pp. 815-820, 2011. 
[9] D. D. Ganji, “The application of He's homotopy perturbation method to nonlinear equations arising in heat transfer," Physics Letters A, vol. 355, no. 4-5, pp. 337-341, 2006.

[10] R. Bellman, Perturbation Techniques in Mathematics, Physics, and Engineering, Holt, Rinehart and Winston, New York, NY, USA, 1964.

[11] J. D. Cole, Perturbation Methods in Applied Mathematics, Blaisedell, Waltham, Mass, USA, 1965.

[12] G. L. Liu, "New research direction in singular perturbation theory: artificial parameter approach and inverse perturbation technique," in Proceedings of the 7th Conference on Modern Mathematics and Mechanics, 1997.

[13] S. J. Liao, The proposed homotopy analysis technique for the solution of nonlinear problems [Ph.D. thesis], Shanghai Jiao Tong University, Shanghai, China, 1992.

[14] V. Marinca, N. Herişanu, and I. Nemeş, "Optimal homotopy asymptotic method with application to thin film flow," Central European Journal of Physics, vol. 6, no. 3, pp. 648-653, 2008.

[15] N. Herişanu and V. Marinca, "Explicit analytical approximation to large-amplitude non-linear oscillations of a uniform cantilever beam carrying an intermediate lumped mass and rotary inertia," Meccanica, vol. 45, no. 6, pp. 847-855, 2010.

[16] V. Marinca, N. Herişanu, C. Bota, and B. Marinca, "An optimal homotopy asymptotic method applied to the steady flow of a fourth-grade fluid past a porous plate," Applied Mathematics Letters, vol. 22, no. 2, pp. 245-251, 2009.

[17] N. Herisanu, V. Marinca, T. dordea, and Gh. Madescu, "A new analytic approach to nonlinear vibration of an electrical machine," Proceeding of the Romanian Academy, vol. 9, pp. 229236, 2008.

[18] V. Marinca and N. Herişanu, "Determination of periodic solutions for the motion of a particle on a rotating parabola by means of the optimal homotopy asymptotic method," Journal of Sound and Vibration, vol. 329, no. 9, pp. 1450-1459, 2010.

[19] H. Ullah, S. Islam, M. Idrees, and M. Arif, "Solution of boundary layer problems with heat transfer by optimal homotopy asymptotic method," Abstract and Applied Analysis, vol. 2013, Article ID 324869, 10 pages, 2013.

[20] M. Idrees, S. Islam, S. I. A. Tirmizi, and S. Haq, "Application of the optimal homotopy asymptotic method for the solution of the Korteweg-de Vries equation," Mathematical and Computer Modelling, vol. 55, no. 3-4, pp. 1324-1333, 2012.

[21] R. Nawaz, S. Islam, I. A. Shah, M. Idrees, and H. Ullah, “Optimal homotopy asymptotic method to nonlinear damped generalized regularized long-wave equation," Mathematical Problems in Engineering, vol. 2013, Article ID 503137, 13 pages, 2013.

[22] S. Iqbal, M. Idrees, A. M. Siddiqui, and A. R. Ansari, "Some solutions of the linear and nonlinear Klein-Gordon equations using the optimal homotopy asymptotic method," Applied Mathematics and Computation, vol. 216, no. 10, pp. 2898-2909, 2010.

[23] M. Idrees, S. Islam, S. Haq, and G. Zaman, "Application of the optimal homotopy asymptotic method to squeezing flow," Computers and Mathematics with Applications, vol. 59, pp. 3858-3866, 2010.

[24] R. Nawaz, H. Ullah, S. Islam, and M. Idrees, "Application of optimal homotopy asymptotic method to Burger equations," Journal of Applied Mathematics, vol. 2013, Article ID 387478, 8 pages, 2013. 


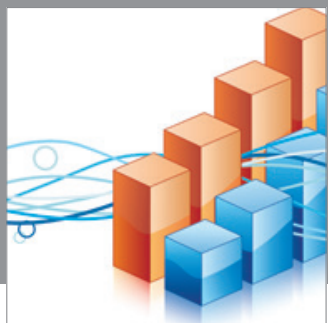

Advances in

Operations Research

mansans

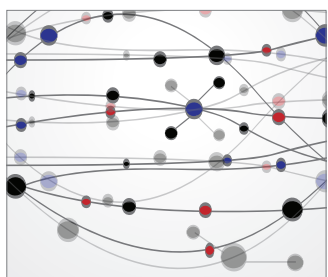

The Scientific World Journal
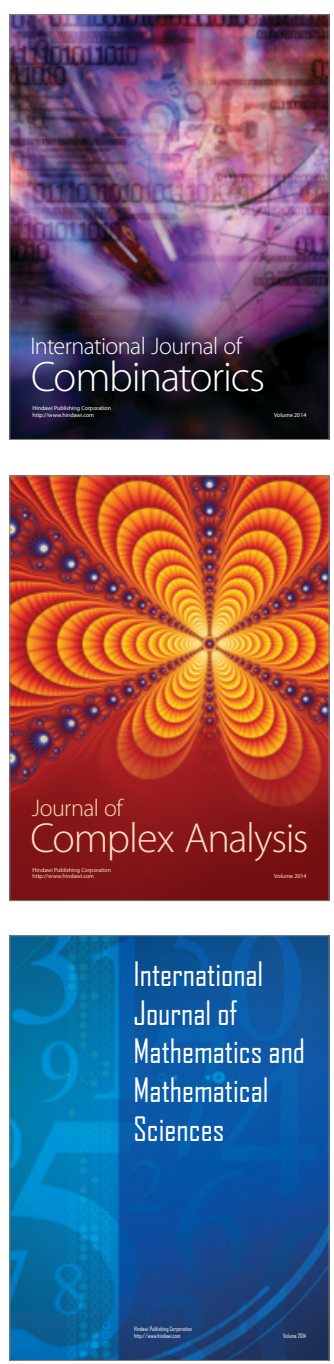
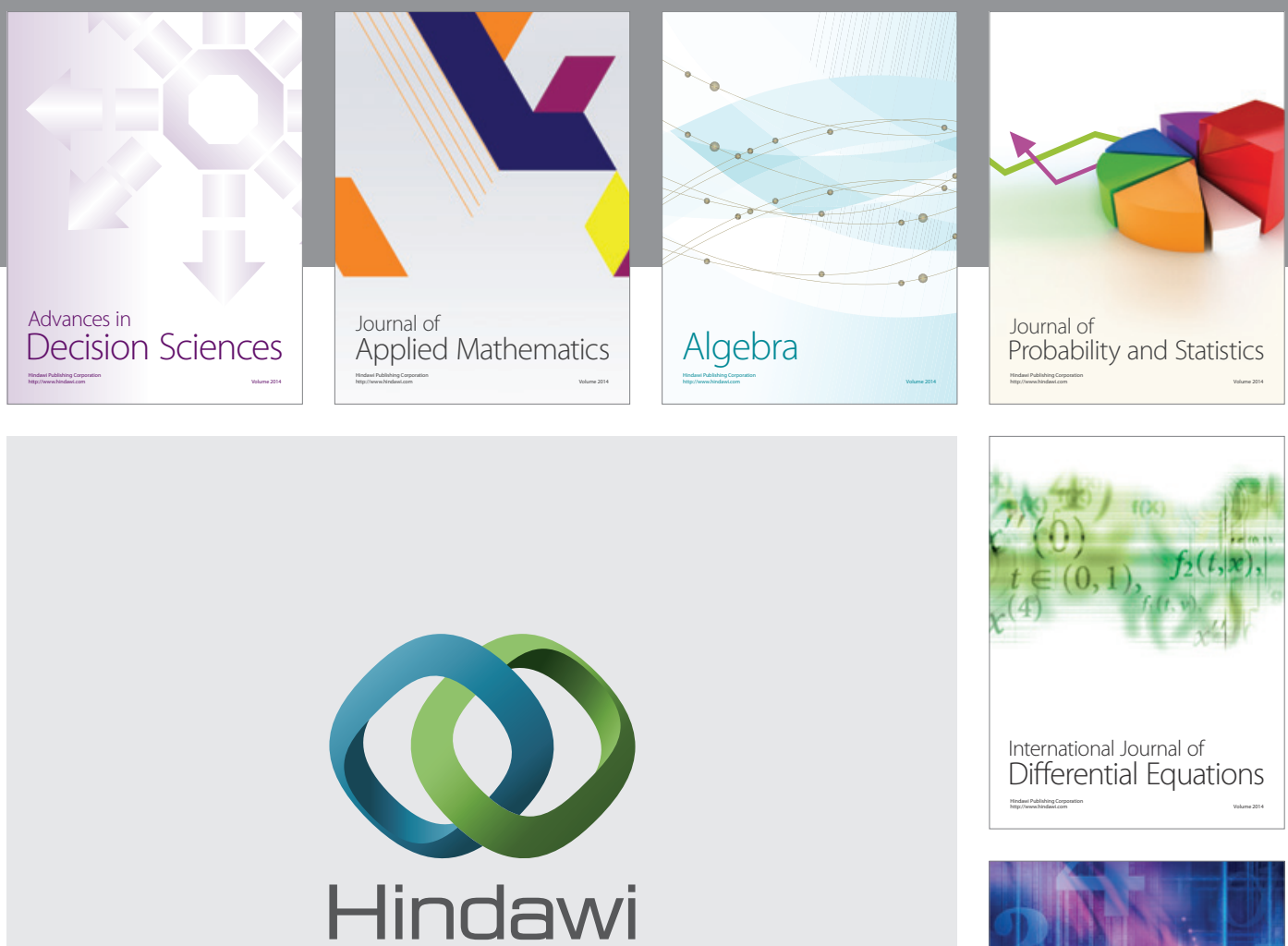

Submit your manuscripts at http://www.hindawi.com
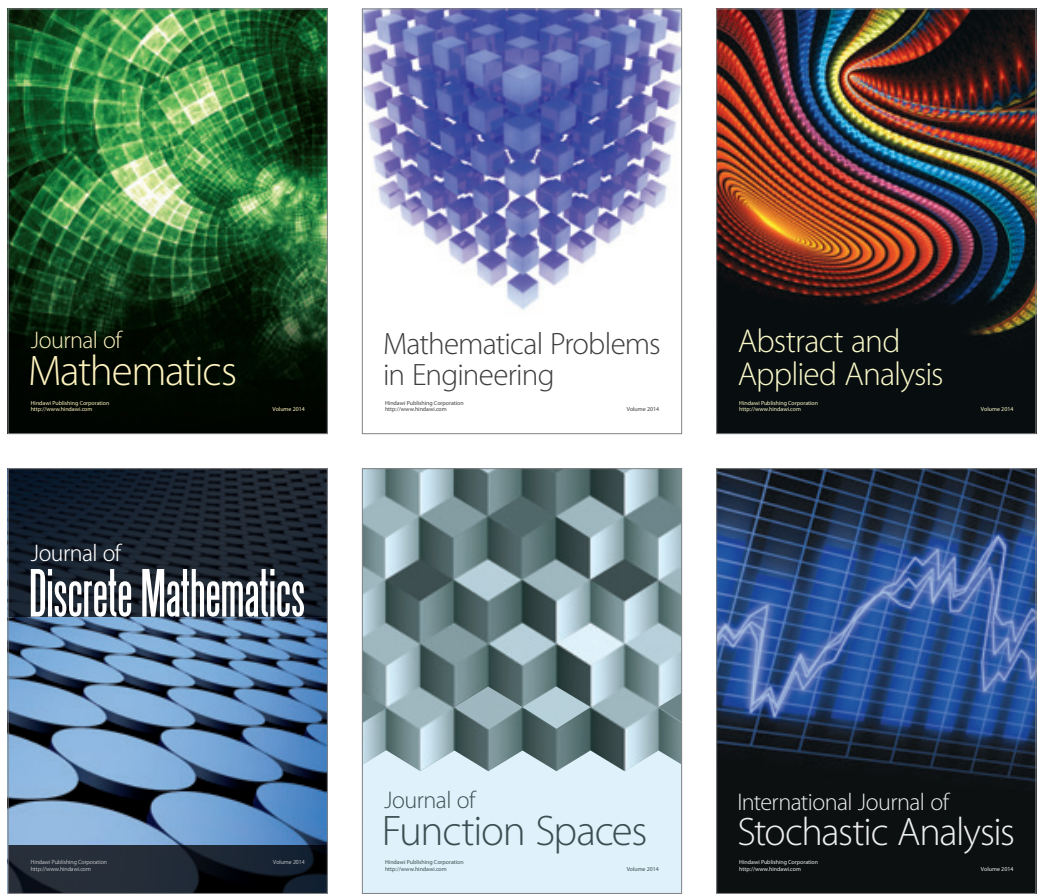

Journal of

Function Spaces

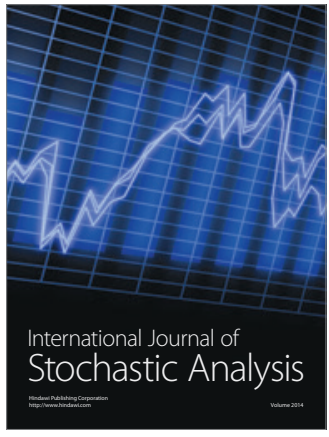

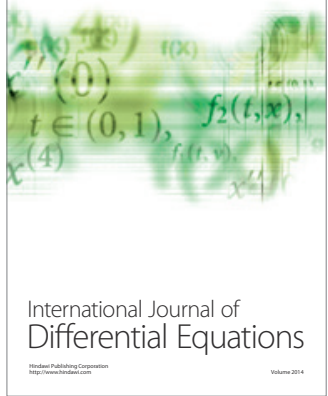
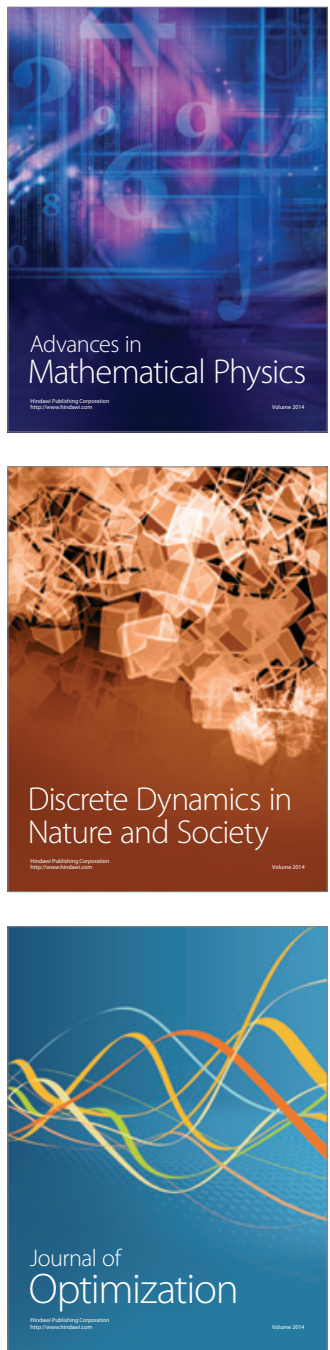